\title{
Effect of Telenursing on the Self-efficacy of Patients with Myocardial Infarction: A Quasi-experimental Study
}

\author{
Najmeh Keshavaraz ${ }^{1}$, Mahin Naderifar ${ }^{2, *}$, Mohammadreza Firouzkohi ${ }^{2}$, \\ Abdolghani Abdollahimohammad ${ }^{2}$, Majid Reza Akbarizadeh ${ }^{3}$
}

\author{
${ }^{1}$ Student Research Committee, Faculty \\ of Nursing and Midwifery, Zabol \\ University of Medical Sciences, Zabol, \\ Iran \\ ${ }^{2}$ Department of Nursing, Zabol \\ University of Medical Sciences, Zabol, \\ Iran \\ ${ }^{3}$ Department of Pediatrics, Zabol \\ University of Medical Sciences, Zabol, \\ Iran
}

*Correspondence

hresearchh@gmail.com

(Mahin Naderifar)

\begin{abstract}
Background and Objective: Despite medical advances, patients with Myocardial Infarction do not fully recover and require rehabilitation and other treatment measures as well. One way to empower these patients is to promote self-efficacy. Therefore, the present study aimed to determine the effect of Tele-nursing on the self-efficacy of patients with myocardial infarction. Methods: This quasi-experimental study was conducted on 40 patients with Myocardial Infarction. The participants selected using the simple random sampling method. The patients were divided into two intervention and control groups of 20 participants. Routine training was given to both groups before discharge. The demographic data questionnaire and Sullivan's cardiac self-efficacy questionnaire were then provided to each patient. In the intervention group, in addition to routine training, the telephone follow-up intervention was performed by the researcher within one month (once a week with an average call duration of 10 minutes). After one month, the self-efficacy questionnaire was provided to each of the study units again. Finally, after determining the normal distribution, the data were analyzed by chi-square, independent t-test, and ANCOVA tests, using SPSS v.25 software. Results: There was no statistically significant difference between the two groups in terms of demographic variables and disease characteristics. The mean score of self-efficacy before and after the intervention in the control group was $22.90 \pm 3.93$ and $33.35 \pm 8.36$, respectively, and in the intervention group, was $25.60 \pm 6.90$ and $47.45 \pm 5.60$, respectively. There was a statistically significant difference between the two groups after the intervention $(p<0.001)$. Conclusion: Telenursing can improve adherence to the treatment program and promote patients' self-efficacy. Therefore, due to its reliability, availability, and low cost, this method can be used in patient care and follow-up.
\end{abstract}

\section{Keywords}

Telenursing, Self-Efficacy, Myocardial Infarction

\section{Introduction}

Acute myocardial infarction (AMI) is one of the most common heart diseases diagnosed in developing countries [1]. The Myocardial Infarction (MI), also known as heart attack, occurs when coronary blood flow is suddenly reduced by the blockage of a coronary artery that has already narrowed due to atherosclerosis. The incidence rate of MI increases progressively with age and the presence of other risk factors such as hypertension, smoking, and diabetes [2]. About 1.5 million people in the United States have suffered from MI in a year, and MI is associated with $30 \%$ mortality rate. In Iran, these diseases are also the first leading cause of death in individuals over 35 years of age [1]. Despite rapid diagnostic and therapeutic advances, one-third of patients with MI still die, and two-thirds of those who survive do not fully recover. Thus, this disease imposes a huge cost on the country's health care systems [3]. On the other hand, more than $50 \%$ of postMI patients require rehabilitation and other treatment measures [4].

The ideal treatment for ischemic heart disease involves the use of multilateral pharmaceutical measures, surgery, controlling risk factors, and lifestyle modification, which leads to the empowerment of these patients. One way to empower these patients is to promote self-efficacy [5]. According to Albert Bandura, self-efficacy is "one's belief in one's ability to succeed in specific situations or accomplish a task" [6]. Health behaviors are strongly affected by self-efficacy, and individuals seem to be stimulated to perform health behaviors and even show health behaviors as confronting challenges when they feel a sense of control over their health behaviors [7]. In other words, self-efficacy is directly associated with health behaviors and for attaining goals, health behaviors are indirectly affected by self-efficacy. Studies have shown 
TA B L E 1. Frequency distribution of individual and clinical characteristics in both the control and the intervention groups.

\begin{tabular}{|c|c|c|c|c|}
\hline \multicolumn{2}{|c|}{ Variable } & \multicolumn{2}{|c|}{ Number (Percentage) } & \multirow[t]{2}{*}{ Significance level } \\
\hline & & Intervention & Control & \\
\hline \multirow[t]{2}{*}{ Gender } & Female & $7(35)$ & $4(20)$ & 0.288 \\
\hline & Male & $13(65)$ & $16(80)$ & \\
\hline Marital status & Married & $20(100)$ & $20(100)$ & 1 \\
\hline \multirow[t]{2}{*}{ Residency } & Village & $9(45)$ & $7(35)$ & 0.519 \\
\hline & City & $11(55)$ & $13(65)$ & $Z=0.417$ \\
\hline \multirow[t]{2}{*}{ Insurance } & Without insurance & $3(15)$ & 0 & 0.115 \\
\hline & With insurance & $17(85)$ & $20(100)$ & $Z=3.24$ \\
\hline \multirow[t]{4}{*}{ Education } & Primary & $11(55)$ & $15(75)$ & \\
\hline & Middle & $7(35)$ & $1(5)$ & \\
\hline & High-school diploma & $1(5)$ & $2(10)$ & 0.105 \\
\hline & Bachelor's degree & $1(5)$ & $2(10)$ & $Z=5.82$ \\
\hline \multirow[t]{4}{*}{ History of the disease } & Without history & $6(30)$ & $5(25)$ & 0.723 \\
\hline & With history & $14(70)$ & $15(75)$ & $Z=0.12$ \\
\hline & Employee & $2(10)$ & $2(10)$ & \\
\hline & Self-employed & $11(55)$ & $13(65)$ & 0.77 \\
\hline \multirow[t]{2}{*}{ Occupation } & Housewife & $6(30)$ & $3(15)$ & $Z=1.67$ \\
\hline & Unemployed & $1(5)$ & $2(10)$ & \\
\hline
\end{tabular}

that a sense of low self-efficacy is characterized by anxiety and discomfort, depression, and psychosomatic symptoms [8]. Individuals with high levels of self-efficacy usually identify difficult tasks as challenges to mastering the situations that arise and do not avoid them [9]. Self-efficacy is a valuable tool for nurses in health care centers, and evaluating patients' self-efficacy by nurses and promoting it can increase patients' motivation to take care of themselves, and also increase life expectancy, and are associated with controlling symptoms, treatment, physical consequences, and psychosocial impacts in patients with chronic diseases [10].

In this regard, health care providers, including nurses, can help patients such as those with MI through developing counseling and training programs [11]. Telenursing provides patients with distance education. Since patients are responsible for taking care of themselves at home after discharge and cannot get the desired treatment without receiving the necessary guidance, telenursing allows them to confront these problems [12]. Telenursing is the application of telecommunication technology in the improvement of patient care, which increases the relationship between the patient and the nurse [13]. The use of this technology provides patients with rapid access to better services and fully improves the quality of health care services provided to patients. Post-discharge phone calls contribute to identifying and correcting care gaps that may occur after discharge from the hospital [14]. It is also used as an appropriate tool for exchanging information, providing health education and awareness, managing the signs and symptoms of the disease and quickly diagnosing the complications, and reassuring patients and their family [15]. The results of studies conducted on patients with cardiovascular disorders have also revealed that telenursing is a reliable, low-cost method to educate patients and follow-up their care, which increases adherence to treatment plans in developing countries such as Iran [16]. Therefore, this study aimed to investigate the effect of telenursing on the self-efficacy of patients with MI.

Study question: what is the effect of telenursing on the selfefficacy of patients with MI?

\section{Methods}

\subsection{Study design and setting}

This pre- and post-test quasi-experimental study was conducted on 40 patients with MI who referred to Amir AlMomenin Hospital from February 1, 2019 to August 10, 2019. The study setting was cardiac wards in one educational hospital of Zabol university of medical science in Zabol city, Iran. In this university the ratio of educators to students in this is 0.7 . The teaching methods are speech and using the PowerPoint slides and $\mathrm{Q} / \mathrm{A}$ with students. The samples were selected using the simple random sampling method and divided into two intervention and control groups of 20 patients.

\subsection{Instruments}

The data collection instruments included demographic data questionnaire (age, gender, residency, marital status, education level, health insurance status, employment status, history of other diseases, duration of the disease, and duration of the treatment) and Sullivan's cardiac self-efficacy questionnaire which consists of 16 questions scored on a 5-point Likert scale ranging from zero (not at all confident) to four (completely 
confident). In this questionnaire, 33 - 64 scores reflect high self-efficacy, 23 - 32 scores indicate moderate self-efficacy, and $0-22$ scores represent low self-efficacy. In the study of Varaei et al. (2014), the validity and reliability of the questionnaire were evaluated and confirmed. The content validity index (CVI) of the questionnaire was $93.4 \%$ for relevance, $98.8 \%$ for clarity, and $90.8 \%$ for simplicity and fluency. The reliability of the questionnaire was measured using internal consistency. The questionnaire was given to 30 patients who were candidates for the coronary artery bypass grafting (CABG) surgery, and the reliability of internal consistency measured using cronbach's alpha. The cronbach's alpha was $0.97[16]$.

\subsection{Data collection}

After the research plan was approved in the Research Council of the School of Nursing and the official permission of the Vice Chancellor for Research was obtained, the researcher went to the hospital to conduct the research. The objectives of the study were first explained to the patients who met the inclusion criteria. Then, each questionnaire, including demographic data questionnaire and Sullivan's cardiac self-efficacy questionnaire, was provided to each patient in the intervention and control group. Routine training was given to both groups before discharge. In the intervention group, in addition to patient training, the telephone follow-up intervention was performed by the researcher for one month [18] so that the telephone follow-up was performed once every week and the average call duration (ACD) was 10 minutes. During the telephone call, the patients were taught about diet, fluid intake, medication regimen, activity and rest, follow-up treatment, and reduction of anxiety and stress. The researcher's contact number was also provided to patients to answer their questions. In the end, Sullivan's cardiac self-efficacy questionnaire was given to each of the study units in the intervention group again. In the control group, without performing any training intervention, the cardiac self-efficacy questionnaire was provided to patients after one month and compared with the intervention group. The all basic life support (BLS) instructors was professional and certified.

\subsection{Statistical analysis}

The data were analyzed using SPSS25 and after the normal distribution was determined using the Shapiro-Wilk test and chi-square, independent t-test and ANCOVA statistical tests, the level of statistical significance was set at $p=0.05$.

\section{Results}

The findings showed that most of the participating units in both the intervention and control groups were male, married, living in the city, had insurance, had primary education, and were self-employed. The majority of patients also had a history of underlying diseases such as diabetes, hypertension, and hyperlipidemia, and was self-employed (Table 1).

According to the independent t-test, no statistically significant difference was observed between the two groups in terms of age $(p=0.906)$, duration of the disease $(p=0.831)$ and duration of the treatment $(\mathrm{p}=0.526)$ variables $(\mathrm{p}>0.05)$ (Table 2).

The independent t-test did not show a significant difference in the self-efficacy scores of the two control and intervention groups before the intervention $(p=0.137)$. The mean score of self-efficacy before and after the intervention in the control group was $22.90 \pm 3.93$ and $33.35 \pm 8.36$, respectively, and in the intervention group, was $25.60 \pm 6.90$ and $47.45 \pm 5.60$, respectively. There was a statistically significant difference between the two groups after the intervention $(p<0.001)$. The ANCOVA test also showed a statistically significant difference in the mean scores after the intervention through controlling the pre-test variable $(\mathrm{p}<0.001)$ (Table 3$)$.

\section{Discussion}

The findings revealed that the average age of patients with MI was 54 years. In Hatami et al.'s study, the average age of patients with MI was reported to be 54 years [19], which is consistent with the results of the present study. In the studies by Rahimi (2019) [20] and Faraji (2015) [21], the average age of patients with MI was 58 years. Also, in the review study by Rahimizadeh et al. on patients with MI in Iran (2017), a higher average age (62.4) was reported [22]; while in the present study, the average age of MI was lower. The reason for this discrepancy may reside in the different lifestyles of the people living in this area, and on the other hand, the scope of the study and the number of participants were regarded as other factors affecting such discrepancy.

According to the results of the present study, most of the patients with MI in both the intervention and the control groups were male, which is consistent with the results of other studies such as Rahimi's [20] and Akbari's [6] study. The results showed that the mean score of self-efficacy in the control group and telephone follow-up intervention group (telenursing) before the intervention was $22.90 \pm 3.93$ and $25.60 \pm 6.90$, respectively, which indicates low and moderate self-efficacy in patients with MI. After the intervention, the mean self-efficacy score increased in both the control group and the telephone follow-up intervention group, which was significantly higher in the intervention group compared to the control group. This positive effect could be due to the appropriate length of intervention in the current study and appropriate telephone training based on each patient's needs (telenursing). The literature review demonstrated that there have been no direct studies of the effect of telenursing on the self-efficacy of patients with MI but similar studies have confirmed the findings of the present study. For instance, Yan et al.'s study (2013) on the effect of telephone follow-up on disease perception and lifestyle following MI in China reported that telephone followup effectively increased positive disease perceptions and that the patients in the intervention group were also more optimistic about their ability to fight the disease, and on the other hand, telephone follow-up interventions improved patients' nutrition and physical activity [23]. In the present study, as in Yan et al.'s study, the telephone follow-up intervention had also positive impacts and effectively increased the self-efficacy of patients with MI. These findings are in line with the results of Najafi et al.'s study (2016). In the study by Najafi et 
TA B L E 2. Mean and standard deviation of age, duration of the disease, and duration of the treatment variables in both the control and the intervention groups.

\begin{tabular}{|lccccc|}
\hline Variable & \multicolumn{2}{c}{ Mean (Standard deviation) } & $\mathbf{9 5 \%}$ confidence limits & Significance level \\
\hline Age & Intervention & Control & & \\
\hline & $54.80(6.73)$ & $54.50(9.05)$ & $-4.80,5.40$ & 0.906 \\
\hline Duration of the disease & $4.42(1.86)$ & $4.26(2.15)$ & $-1.37,1.70$ & $\mathrm{t}=0.119$ \\
\hline & & & & 0.831 \\
\hline Duration of the treatment & $4.42(1.86)$ & $3.93(2.25)$ & $-1.07,2.07$ & 0.521 \\
\hline
\end{tabular}

T A B L E 3. Mean and standard deviation of self-efficacy scores before the intervention, after the intervention, and in the modified state in both the control and the intervention groups.

\begin{tabular}{|cccccc|}
\hline Self-efficacy & \multicolumn{2}{c}{$\begin{array}{c}\text { Mean (Standard deviation) or mean } \\
\mathbf{( 9 5 \%} \text { confidence limits) }\end{array}$} & 95\% confidence limits & Significance level \\
\hline & Intervention & Control & & \\
\hline Before the intervention & $25.60(6.90)$ & $22.90(3.93)$ & $-0.89,6.29$ & 0.137 \\
\hline & & & & $\mathrm{t}=1.52$ \\
\hline After the intervention & $47.45(5.60)$ & $33.35(8.36)$ & $18.65,9.54$ & $<0.001$ \\
\hline & & & & $\mathrm{t}=6.26$ \\
\hline In the modified state & 46.5 & 34.29 & $-16.16,-8.26$ & $<0.001$ \\
\hline & $(43.74,49.26)$ & $(31.53,37.06)$ & & $\mathrm{F}=38.94$ \\
\hline
\end{tabular}

al. regarding the effect of nurse-led telephone follow-ups on adherence to dietary and medication regimens in postMI patients, the results revealed that there was a statistically significant difference between the two intervention and control groups after the intervention, and the results also emphasized the positive effects of nurse-led telephone follow-ups. In this study, it is recommended that health care providers, especially nurses, apply telenursing to patient self-care [24]. The study by Farazmand et al. (2016) is another study that is consistent with the results of the present study. In this study, that aimed to determine the effect of telephone follow-up after discharge on the level of hope and the quality of life of patients admitted to a cardiac care unit (CCU), the results demonstrated that hope and quality of life were significantly improved in the telephone intervention group [18]. Javanmard et al.'s study (2017), which aimed to determine the effect of telenursing on self-efficacy in patients with non-alcoholic fatty liver disease (NAFLD), showed that the mean scores of nutritional selfefficacy and physical activity in the intervention group were significantly improved after the intervention [25]. In the study by Mohammadi et al. (2016) regarding the effect of nurseled telephone follow-ups on the self-efficacy of women with type 2 diabetes, the results revealed that self-efficacy increased significantly in the experimental group [26]. In a study by Shojaei and colleagues entitled "A comparative study of the effect of patient education and nurse-led telephone follow-up on the level of anxiety in patients with heart failure", it has been found that the telephone follow-up intervention reduces patient anxiety more effectively [27]. On the other hand, in Imeh et al.'s study (2014), that investigated the effect of telemedicine-based virtual admission on the self-efficacy of patients with chronic obstructive pulmonary disease (COPD), no statistically significant difference was observed in the selfefficacy scores of the two groups after the intervention, which is inconsistent with the results of the current study. It appears to be due to different research communities and different conditions associated with the disease [28].

The impact of telenursing on patients' self-efficacy in the present study and other studies could be due to the fact that the use of telenursing technology increases communication between the patient and the nurse and provides the patient with quick access to specialized services and skills, and also continuously following up patients allows them to gradually acquire the ability to manage and handle the complications and symptoms of the disease and to exhibit appropriate health behaviors when encountering changing circumstances. It appears that telenursing can play an effective role in helping patients better adapt to the disease complications and in improving their performance through increasing self-efficacy. In fact, changing self-efficacy is a basic prerequisite for proper selfmanagement during the recovery period of patients with heart disease [29].

\section{Conclusion}

The results of this study indicated that training provided by nurses and nurse-led telephone follow-ups increased the selfefficacy of patients with MI. This method can increase selfefficacy due to lack of time and space constraints, lack of need to commute and reduction of additional costs as well as timely access to information about and training in care tips. Therefore, it is recommended to take nurse-led telephone follow-ups 
(telenursing) into consideration in health care centers.

\section{ACKNOWLEDGMENTS}

This article is the result of a master's thesis in medical-surgical nursing. I would like to express my gratitude and appreciation to the esteemed Vice Chancellor for Research of Zabol University of Medical Sciences and all nursing staff of Amir AlMomenin Hospital in Zabol and those who participated in the study for their assistance with the research project.

\section{CONFLICT OF INTEREST}

We declare that we do not have any commercial or associative interest that represents a conflict of interest in connection with the work submitted.

\section{ETHICAL CONSIDERATIONS}

This study was approved by the Ethics Committee of Zabol University of Medical Sciences (Ethic code: IR.ZBMU.REC.1397.213). Before beginning the study, the consent of relevant authorities was obtained. The written and oral consent were obtained from participants.

\section{REFERENCES}

[1] Rejeh N, Heravi-Karimooi M, Taheri Kharame Z, et al. Quality of life in patients with myocardial infarction and related factors: A cross sectional Study. IJNR. 2015;9:1-11.

[2] Kaibab Z, Mirzaei M, Dabiran S. The Pattern of Age Changes of First Acute Myocardial Infarction in Rafsanjan During 2003 to 2012: Has Age Decreased? JRUMS. 2017;15:1015-1026.

[3] Shahsavari S, Nazari F, Karimyar Jahromi M, et al. Epidemiologic study of hospitalized cardiovascular patients in Jahrom hospitals in 2012- 2013. Cardiovascular Nursing Journal. 2013;2:14-21.

[4] Vahedian-azimi A, Alhani F, Goharimogaddam K, et al. Effect of family - centered empowerment model on the quality of life in patients with myocardial infarction: A clinical trial study. 3 JNE. 2015;4:8-22.

[5] Boroumand S, Shahriari M, Abbasi Jebeli M, et al. Determine the level of self-efficacy and its related factors in patients with ischemic heart disease: A descriptive correlational study. IJNR. 2015;9:61-69.

[6] Akbari O, Vaghar Seyedin SA, Saadatjoo SA, Kazemi T. Effect of Continuous Care Model on the Self-Efficacy of Patients with Myocardial Infarction in Controlling Disease Complications. MedicalSurgical Nursing Journal. 2015;3:185-194.

[7] Zand S, Rafiei M. The efficacy of an instrument designed for specific self - efficacy measurment in patients with mi. Nurs Midwifery J. 2013;11:00 .

[8] Behnam Moghadam M, Behnam Moghadam A, Yarian S, et al. Predicting the Quality of Life Based on Public Health, Social Support and Self Efficacy in Cardiovascular Patients. Armaghane danesh. 2014;19:633642.

[9] Sanaie N, Nejati S, Zolfaghari M, et al. The Effects of Family-based Empowerment on Family Cooperation in Following Patient Treatment Regime after Coroner Arteries Bypass Surgery. Mod Care J. 2014;11:1927.

[10] Baljani E, Rahimi J, Amanpour E, et al. Effects of a Nursing Intervention on Improving Self-Efficacy and Reducing Cardiovascular Risk Factors in Patients with Cardiovascular Diseases . Hayat. 2011;17:45-54.

[11] salavati M, khatiban M, moghadari koosha B, et al. Evaluating the Effect of Teach Back Education on Self-Care Behaviours and Quality of Life in Patients With Myocardial Infarction in 2015: A Randomised Controlled Trial. Sci J Hamadan Nurse Midwifery Fac. 2017;25:1-8.
[12] Elham M, Samadi N, Mehrnush N, et al. Tele Nursing: Optimal Option for Patient Educational Improvement. JHC. 2011;13:0-0.

[13] Kamrani F, Nikkhah S, Borhani F, et al. The effect of patient education and nurse-led telephone follow-up (telenursing) on adherence to treatment in patients with acute coronary syndrome. Cardiovascular Nursing Journal. 2015;4:16-24.

[14] Forouzesh M, Sanagoo A, ghavidel A, et al. The effect of telenursing (telephone follow up) after discharge on readmission due to complications after coronary artery bypass graft surgery. J Urmia Nurse Midwifery Fac. 2017; 15:584-594.

[15] Raziani F, Naderipour A, Jalali R, et al. The effect of duration of using cochlearimplant and hearing aid on the speech intelligibility of children with hearing loss. J Clin Res Paramed Sci. 2017;6:300-309.

[16] Shirkosh S, Rostami M. Telenursing: A new opportunity for care of chronic patients. cjhaa. 2017;1:49-55.

[17] Varaei S, Shamsizadeh M, Cheraghi MA, et al. Effects of a peer education on cardiac self-efficacy and readmissions in patients undergoing coronary artery bypass graft surgery: a randomized-controlled trial. Nursing in critical care. 2017;22:19-28.

[18] Farazmand J, Nasiripour AA, Raeissi P. The Effect of Telephone Followup Programs after Hospital Discharge on Hope and the Quality of Life in Patients Admitted to the Coronary Care Unit (CCU) . JBUMS 2017; 19:41-46.

[19] hatami $\mathrm{H}$, hasanpoor $\mathrm{A}$, salehi $\mathrm{S}$, et al. Investigating the Effect of Intervention Based on Basnef Model on Quality of Life Among Myocardial Infarction Patients. Journal of Clinical Nursing and Midwifery. 2020;8:526-534.

[20] Rahimi H, Azizkhani L, Vahedi S, et al. Study of the Duration, Outcomes, and Related Factors of Reperfusion Therapy in Patients with ST-Segment Elevation Myocardial Infarction. Avicenna J Clin Med. 2020;26:220-226.

[21] faraji N, Pashaeypoor S, negarandeh R. The effect of pre-discharge education and telephone follow-up on illness perception and lifestyle in patients with myocardial infarction. Avicenna $J$ Nurs Midwifery care. 2015;23:82-91.

[22] Rahimzadeh M, Kavehei B. Short-term survival rates after myocardial infractions in Iran: meta-analysis and systematic review. J Arak Univ Med Sci. 2017;20:57- 67.

[23] Yan J, You LM, Liu B, et al. The effect of a telephone follow-up intervention on illness perception and lifestyle after myocardial infarction in China: A randomized controlled trial. Int J Nurs Stud. 2014;51:844-55.

[24] Najaf SS, Shaabani M, Momennassab M, et al. The Nurse-Led Telephone Follow-Up on Medication and Dietary Adherence among Patients after Myocardial Infarction: A Randomized Controlled Clinical Trial. IJCBNM. 2016;4:199-208.

[25] Javanmardifard S, Ghodsbin F, Kaviani MJ, et al The effect of telenursing on self-efficacy in patients with non-alcoholic fatty liver disease: a randomized controlled clinical trial. Gastroenterol Hepatol Bed Bench. 2017;10:263-271.

[26] Mohammadi N, Soleymani R, Omidi A, et al. The Effect of Telephone Nursing Follow-up on Self-Efficacy of Females with Type 2 Diabetes Mellitus. Sci J Hamadan Nurse Midwifery Fac. 2017;25:61-68.

[27] Shojaee A, Nehrir B, Naderi N, et al. Comparison of patient education and follow up by nurse on anxiety in heart failure patients. Medical-Surgical Nursing Journal. 2014;3:52-60.

[28] Emme C, Mortensen EL, Rydahl-Hansen S, et al. The impact of virtual admission on self-efficacy in patients with chronic obstructive pulmonary disease - a randomised clinical trial. J Clin Nurs. 2014;23:3124-37.

[29] Carroll Diane L, Robinson E, Buselli E, et al. Activities of the APN to Enhance Unpartnered Elders Self-Efficacy After Myocardial Infarction. Clin Nurse Spec. 2001;15:60-66.

How to cite this article: Najmeh Keshavaraz, Mahin Naderifar, Mohammadreza Firouzkohi, Abdolghani Abdollahimohammad, Majid Reza Akbarizadeh. Effect of Telenursing on the Self-efficacy of Patients with Myocardial Infarction: A Quasi-experimental Study. Signa Vitae. 2020;16(2):92-96. doi:10.22514/sv.2020.16.0039. 